\section{Brassica-sensible Kinder}

ensibilisierungen gegen die Nahrungsmittelallergene Kuhmilch, Hühnereier, Weizen, Fisch und Soja sind häufig bei Kindern mit atopischer Dermatitis. Finnische Wissenschaftler berichten nun über zwei Neuzugänge für diese Liste: Raps (Brassica napus) und Rübenkohl (Brassica rapa). In einer Kohorte von 1.887 Kindern mit Neurodermitis zeigten knapp 11\% einen positiven Pricktest auf entsprechende Allergenextrakte, in einer kleineren Gruppe von Prick-positiven Kindern verlief die offene Provokation mit Rübenkohl bei fast $90 \%$ ebenfalls positiv. Die Reaktionen waren überwiegend dem Soforttyp zuzuordnen und zeigten sich als Lippenschwellung oder Gesichtsurtikaria. Die Auslösedosierungen variierten stark, Kreuzreaktionen auf beide Brassica-Arten

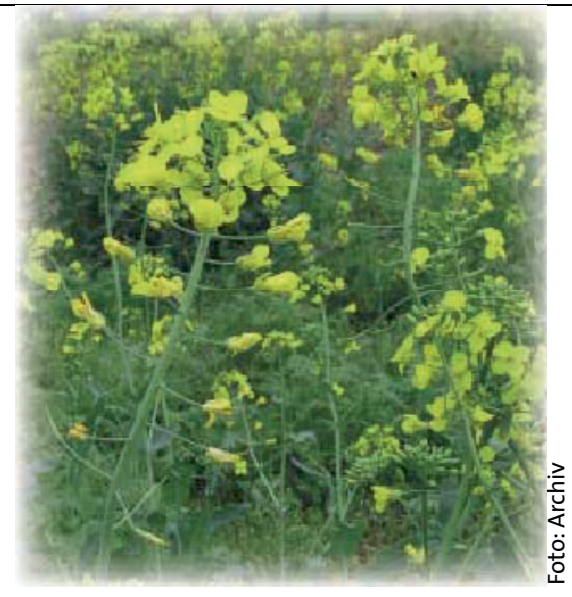

sind wahrscheinlich. Als Allergen kommt ein 2S-Albumin in Frage, das auch das Hauptallergen von Senf (Sinapis alba, Brassicaceae, Kreuzblütengewächse) ist. Eine schlüssige Erklärung für die Zunahme der Brassica-Sensibilisierungen bei den im Durchschnitt vierjährigen Kindern gibt es bisher nicht.

Poikonen S et al. Allergy 2006; 61: 124-7

\section{Entwarnung bei Handys}

eit Herbst 2000 wird im Rahmen der Interphone-Studie in 13 Ländern nach einem Zusammenhang zwischen Gehirntumoren und der Nutzung von Mobiltelefonen gesucht. Die jetzt vorab publizierten Ergebnisse des deutschen Studienzweiges geben weitgehend Entwarnung: Mobiltelefonierer haben kein erhöhtes Risiko, an Gliomen oder Meningeomen zu erkranken. Allein Personen, die seit über zehn Jahren Handys nutzen, weisen ein leicht erhöhtes Risiko für Gliome auf. Statistisch valide wird das Ergebnis allerdings erst mit dem Vorliegen der Daten aus den anderen Studienländern, damit ist Anfang $2007 \mathrm{zu}$ rechnen. es

Schuz J et al Am J Epidemiol 2006; 163:

$512-20$

\section{Warnung vor Zecken}

E n isolierter Lichen planus der Nägel ist selten. Noch seltener dürfte ein isolierter Lichen planus aller 20 Nägel mit starker Aufrauung, ausgeprägter Rillenbildung und distaler Atrophie sein, mit dem ein 51-jähriger Japaner in der Klinik vorstellig wurde. Der Patient litt unter starkem Juckreiz aller Finger- und Fußnägel sowie der Nagelfalze, der übrige Körper, Kopfhaut und Mundschleimhaut waren dermatologisch ohne Befund. Klinik und Histologie ergaben die Diagnose Lichen planus, im Epikutantest wurde eine Re-

aktion gegen Nickel und Gold festgestellt. Als Auslöser rückten nun die Goldfüllungen der Zähne ins Visier. Sie wurden nach vergeblichen Therapieversuchen des Lichen planus mit topischen Glukokortikoiden - schließlich entfernt. Zwei Wochen später verschwanden der Juckreiz und zwölf Wochen später die Nagelfalzschwellungen, nach 24 Wochen wuchsen die Nägel normal geformt nach. $\quad b k$

Yokozeki H et al. Br J Dermatol 2005; 152: 1087-9

\section{Die Allergie der RIP-Forscher}

$\mathrm{V}$ iele Wissenschafter beschäftigen sich aktuell aufgrund deren antiviralen und antiproliferativen Eigenschaften mit Ribosomen-inaktivierenden Proteinen (RIP) - und reagieren dabei manchmal auch allergisch auf den Gegenstand ihrer Forschung. Tatsächlich fanden sich bei vier von 17 italienischen RIP-Forschern allergische Symptome, die durch den Nachweis von spezifischen IgE-Antikörpern auf RIP zurückgeführt werden konnten. Auch ein beschwerdefreier Mitarbeiter zeigte im
ELISA eine positive IgE-Reaktion, was das Vorkommen „stiller" Sensibilisierungen nahelegt. Diese Beobachtungen sollten bei der Entwicklung marktreifer Medikamente auf der Basis von RIP berücksichtigt werden: Wenn fast ein Viertel der Forscher allergische Symptome gegen diese Stoffe zeigt, spricht das nicht für eine besonders gute Verträglichkeit.

$b k$

Szalai K et al. Clin Exp Allergy 2005; 35: 1354-60

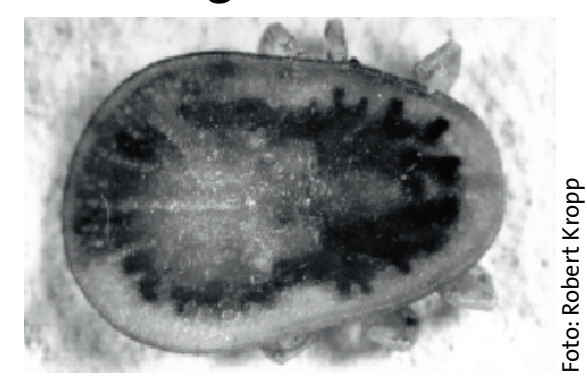

aubenzecken (Argas reflexus) sind nicht wählerisch - im Notfall benutzen sie auch den Mensch als Wirt. Ihre Bisse sind aus allergologischer Sicht allerdings nicht so dramatisch wie befürchtet. Von 148 Leipzigern mit einem Taubenzeckenstich in der Anamnese berichteten $8 \%$ über systemische Reaktionen wie Urtikaria, Angioödem, Dyspnoe oder kardiale Effekte, die übrigen hatten lokale Reaktionen. Eine Atopie erwies sich als Risikofaktor für eine Sensibilisierung, hatte aber keinen Einfluss auf die Schwere der Reaktion. Ein für die Studie hergestellter Zecken-Gesamtextrakt ergab im Pricktest zwar positive Resultate, kann aber für eine allgemeine Diagnostik noch nicht empfohlen werden.

Kleine-Tebbe J et al. J Allergy Clin Immunol 2006; 117: 190-5 